\title{
Habilitadores da transformação digital em direção à Educação 4.0
}

Katyeudo Karlos de Sousa Oliveira - Programa de Pós-Graduação em Informática Aplicada (PPGIA) - Universidade Federal Rural de Pernambuco (UFRPE) karlos.1914.so@gmail.com

Ricardo André Cavalcante de Souza - Departamento de Computação (DC) - Programa de Pós-Graduação em Informática Aplicada (PPGIA) - Universidade Federal Rural de Pernambuco (UFRPE) - ricardo.souza@ufrpe.br

Resumo: À medida que a globalização e os rápidos avanços tecnológicos transformavam o modo de convivência humana e o mundo do trabalho, os sistemas educacionais cresciam desconectados das realidades e necessidades da sociedade e economia global. Entretanto, a pandemia do vírus COVID-19, que provocou a interrupção de aulas em escala mundial, tende a ser um catalisador para a transformação digital na educação. A transformação digital na educação envolve, além do uso de tecnologia, habilidades digitais necessárias a estudantes e professores, e a adoção de pedagogias e práticas inovadoras. Esse trabalho apresenta uma consolidação dos habilitadores organizacionais, tecnológicos e humanos que devem orientar as estratégias de transformação digital em direção à Educação 4.0, resultante da análise de documentos de organizações internacionais de referência.

Palavras-chave: educação 4.0, transformação digital, habilitadores, habilidades.

\section{Digital transformation drivers toward Education 4.0}

\begin{abstract}
As globalization and rapid advancements in technology transformed human coexistence and the world of work, education systems have grew disconnected from the realities and needs of society and global economy. However, the pandemic of the COVID19 virus, which caused the interruption of classes on a global scale, tends to be a catalyst for digital transformation for education. The digital transformation for education involves, in addition to the use of technology, digital skills necessary for students and teachers, and the adoption of innovative pedagogies and practices. This work presents a consolidation of the organizational, technological and human enablers that must guide the strategies of digital transformation toward Education 4.0, resulting from documents analysis produced by international reference organizations.
\end{abstract}

Keywords: education 4.0, digital transformation, enablers, skills.

\section{Introdução}

As escolas necessitam preparar estudantes para trabalhos que ainda não foram criados, para tecnologias que ainda não foram inventadas, para resolver problemas que ainda não foram previstos (OECD, 2018b). O uso produtivo das tecnologias digitais é uma exigência da $4^{\mathrm{a}}$ Revolução industrial iniciada na última década e que está desencadeando a substituição do trabalho mental humano por inteligência artificial, automação e outras inovações digitais (Schwab, 2016).

A Educação 4.0 visa equipar estudantes com as habilidades técnicas, cognitivas, sociais e emocionais necessárias para o aprendizado do século XXI (UNESCO, 2015), e com as competências digitais para atender as exigências dos trabalhos emergentes da $4^{\text {a }}$ Revolução Industrial (WEF, 2020). Para tanto, é necessária uma transformação digital na educação. Transformação digital não é um projeto, é um modo permanente de trabalhar, pensar e agir que busca explorar tecnologias digitais para criar melhor experiência (Baxendale, 2019). Tornar-se digital não é simplesmente usar tecnologias novas, mas também criar mudanças nos processos, pessoas e na cultura de uma organização (Haddud V. $18 \mathrm{~N}^{\mathrm{o}} 1$, julho, 2020 RENOTE DOI: 
e McAllen, 2018). No contexto da educação, a transformação digital envolve repensar os processos de ensino e aprendizado para um público de nativos digitais, considerando os aspectos de fazer digital, por meio de conhecimentos e competências técnicas, e ser digital, por meio da incorporação de habilidades, mentalidade e atitudes digitais.

A transformação digital em direção à Educação 4.0 é uma demanda econômica e social. De acordo com uma estimativa recente, mais de 11,5 trilhões de dólares podem ser adicionados ao PIB global até 2028 se os países forem bem sucedidos em melhor preparar os estudantes para as necessidades da economia do futuro (WEF, 2020). Do ponto de vista social, cerca de 53 milhões de estudantes brasileiros foram impactados de alguma forma por não conseguirem ir fisicamente às escolas devido a pandemia do Covid19 (UNESCO, 2020). As tecnologias digitais nos permitem encontrar respostas inteiramente novas sobre o que as pessoas aprendem, como as pessoas aprendem, onde as pessoas aprendem e quando elas aprendem (OECD, 2020).

Considerando o cenário da pandemia do COVID-19 como ponto de inflexão (tipping point) (Gladwell, 2006) para transformação digital na educação, esse trabalho apresenta um panorama dos habilitadores tecnológicos, humanos, organizacionais e pedagógicos para a transformação digital em direção à Educação 4.0.

\section{Material e Métodos}

Este trabalho visa fornecer uma contribuição teórica, caracterizando-se como uma pesquisa qualitativa, entendida como uma expressão aplicada de modo livre para se referir as pesquisas onde os achados não são sujeitos a quantificação e a algum tipo de análise quantitativa (Minayo et al., 2001). Da perspectiva dos procedimentos técnicos, efetuouse levantamento bibliográfico, isto é, com base em material já publicado sobre o assunto (Gil, 2007). As fontes de informação utilizadas no levantamento dos habilitadores para a transformação digital na educação foram publicações das organizações internacionais: Organisation for Economic Co-operation and Development (OECD), World Economic Forum (WEF), UNESCO, e The United Kingdom Open University. Tais organizações mantêm fóruns permanentes de discussão sobre educação e economia em âmbito mundial.

\section{Resultados e Discussão}

No contex to deste trabalho, habilitadores correspondem a drivers, fatores que dão suporte, habilitam e servem para nortear e direcionar iniciativas de transformação digital em direção à Educação 4.0. A Figura 1 apresenta o panoramageral dos habilitadores mais destacados nas publicações das organizações internacionais utilizadas como referência. Os habilitadores estão agrupados nas seguintes categorias: Tecnológico, envolve recursos, infraestrutura, e abordagens; Organizacional, envolve processos e práticas da instituição educacional; Competência digital docente, envolve as habilidades técnicas, sociais e cognitivas dos professores; Soft skill discente, envolve as habilidades sociais, cognitivas e emocionais dos estudantes; Hard skill discente, envolve as habilidades técnicas e conhecimentos práticos dos estudantes; e Pedagogia, envolve abordagens pedagógicas inovadoras e metodologias ativas. A adoção individual de um habilitador pode trazer benefícios e desafios, porém a combinação entre eles potencializa uma verdadeira transformação digital na educação.

\subsection{Habilitadores Tecnológicos}

Diversas tecnologias transformaram por completo o modo como a sociedade vive e trabalha. Tal transformação não está limitada a uma simples extensão da terceira revolução industrial, representada pelo uso de tecnologias para automatizar a produção de bens e serviços, mas sim para uma era distinta, onde a velocidade da evolução não tem 
precedente histórico, avançando rapidamente e expondo sistemas completos de todos os setores da sociedade (Schwab, 2016). Este fenômeno é consequência do fato de que a sociedade vive em um mundo mais interligado e multifacetado, onde cada vez mais surgem novas tecnologias com constante melhoria do cotidiano (WEF, 2020).

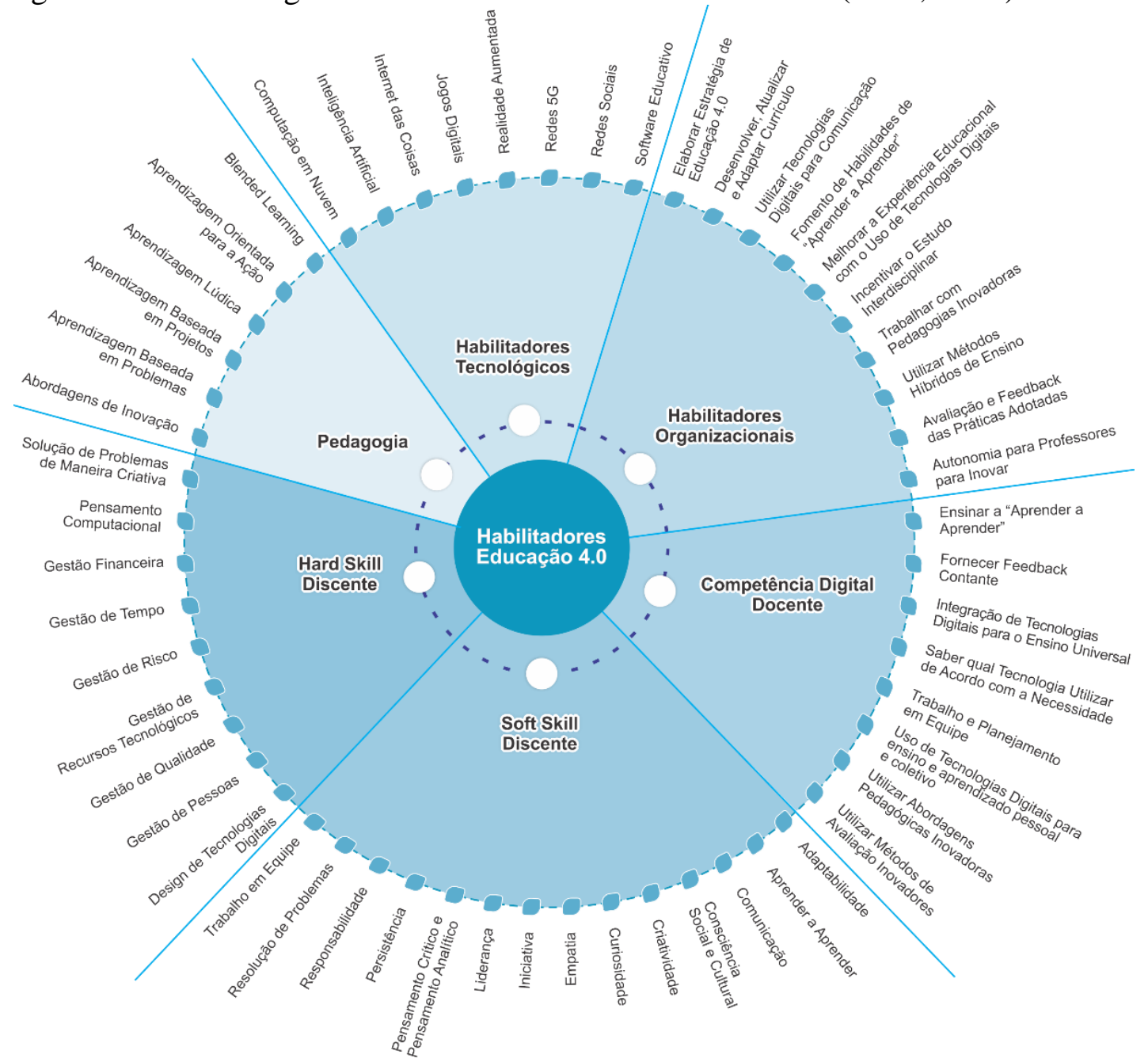

Figura 1 - Habilita dores para transformação digitalem direção à Educação4.0. Fonte: Autores

A seguir é descrito como cada habilitador tecnológico pode contribuir com a transformação digital da educação. Não é apresentada a definição técnica do habilitador, por ser considerada de entendimento comum.

- Computação em Nuvem: Possibilita serviços onde estudantes e professores podem criar e editar documentos, armazenar e compartilhar arquivos, entre outros, sem a necessidade de possuir programas instalados diretamente nas máquinas, permitindo o trabalho colaborativo nos mesmos documentos em ambientes diferentes e contribuição com o trabalho do outro (WEF, 2020).

- Inteligência Artificial: Possibilita o desenvolvimento de sistemas de tutoria inteligente, adequando exercícios e avaliações conforme cada perfil, viabilizando um aprendizado individualizado e personalizado. Permite ainda, com chatbots, a oferta de informações instantâneas aos estudantes ou recomendar como eles podem utilizar o tempo livre mais produtivamente (Kukulska-Hulme et al., 2020).

- Internet das Coisas: Vários objetos dentro do ambiente da sala de aula podem ter dispositivos embarcados que trocam informações entre si, se comunicando com dispositivos que os professores ou os alunos controlam, podendo se conectar com V. $18 \mathrm{~N}^{\mathrm{o}} 1$, julho, 2020 RENOTE DOI: 
várias outras instituições de ensino de diversos níveis, evidenciando parcerias e cooperação entre essas instituições (OECD, 2019).

- Jogos Digitais: Pode assumir distintas vertentes, como gamificação e serious games (jogos sérios). Em contextos educacionais, é capaz de agregar parte da capacidade psicológica, emocional e social dos jogos (OECD, 2018a).

- Realidade Aumentada: Pode incrementar mais um desafio para soluções de problemas, movendo os alunos para novas geografias de modo virtual fora de seus contextos já conhecidos (WEF, 2020).

- Redes 5G: Possibilita aos alunos adotar o conceito de educação digital e aprender sem restrição de espaço e tempo em vários formatos. Possibilita novas modalidades de aprendizagem (transmissões ao vivo e realidade virtual), habituando o estudante a incluir o aprendizado integrado às rotinas diárias (Tam e El-Azar, 2020).

- Redes Sociais: Auxilia na informação e organização dos estudantes sobre questões globais. Essas interações têm uma capacidade poderosa no entendimento do mundo pelos alunos e também auxiliam no desenvolvimento de empatia, primordial para apoiar um mundo mais inclusivo (WEF, 2020).

- Software Educativo: Possibilita avaliações com feedback imediato. Possibilita que as informações sejam comparadas e organizadas. Permite, por exemplo, a orientação e o uso do estudo de idiomas e compreensão de atividades relacionadas, através da informação visual e auditiva de som e imagem (OECD, 2018a).

\subsection{Habilitadores Organizacionais}

A tecnologia e a globalização estão alterando os modelos de negócios em todos as esferas, acelerando o desaparecimento de alguns empregos e a promoção de outros, incluindo novos modos de trabalho, bem como o desenvolvimento de habilidades para os empregos existentes. Os sistemas educacionais, que seguiram praticamente estáticos por décadas, são em sua maioria inadequados para essas novas necessidades (WEF, 2017). Espera-se que os sistemas educacionais exerçam diversas tarefas relevantes de forma simultânea, inclusive influenciando os jovens como futuros cidadãos, e também auxiliando-os a desenvolver conhecimentos e habilidades fundamentais para atingir um melhor êxito no trabalho e na vida (Peterson et al., 2018).

A escola deve se ajustar para equipar as pessoas com as habilidades necessárias para criar um mundo mais inclusivo, coeso e produtivo (OECD, 2018b). As escolas necessitam passar por uma reestruturação organizacional, considerando como principais habilitadores: Elaborar e possuir uma estratégia de Educação 4.0 (Schleicher, 2016; OECD, 2018b); Desenvolver, atualizar e adaptar continuamente o currículo escolar (Schleicher, 2016; WEF, 2017, 2020); Utilizar tecnologias digitais no suporte a comunicação, retirando restrições de espaço e tempo entre as pessoas, inclusive com outras escolas (WEF, 2017); Concentrar o fomento de habilidades no ensino do "aprender a aprender" (WEF, 2017, 2020; OECD, 2018b); Melhorar a experiência educacional com o uso de tecnologias digitais no cotidiano dos alunos (Paniagua e Istance, 2018; OECD, 2018b); Incentivar o estudo interdisciplinar (WEF, 2017; Paniagua e Istance, 2018); Trabalhar com pedagogias inovadoras (Paniagua e Istance, 2018); Utilizar métodos híbridos de ensino (EAD, semipresencial, etc.) (Paniagua e Istance, 2018); Avaliação e feedback constantes das práticas aplicadas aos professores e estudantes (Schleicher, 2016; OECD, 2018a); e Dar aos professores a autonomia e suporte para que eles possam inovar na sala de aula (Schleicher, 2016; OECD, 2018a).

\subsection{Competência Digital Docente}


A educação outrora era basicamente sobre ensinar algo para às pessoas; atualmente, é necessário assegurar que os estudantes desenvolvam habilidades que os orientem no seu próprio caminho em ambientes cada vez mais incertos, voláteis e ambíguos (OECD, 2018b, 2019). Os professores precisam ser também equipados com competências digitais, envolvendo conhecimento tecnológico e adoção de mentalidade e atitude digitais. Os habilitadores para a transformação digital docente consistem de:

- Ensinar a "aprender a aprender": Estimular a autonomia e responsabilidade, através de, por exemplo, oportunizar que os alunos possam ajudar nas regras e atividades na sala de aula. Ensinar habilidades de argumentação para que os alunos possam explicar seus pensamentos, se colocando em um processo colaborativo de descoberta e discussão (OECD, 2018a; Paniagua e Istance, 2018);

- Fornecer feedback constante: Utilizar tecnologias como, redes sociais, wikis e documentos online, para possibilitar a interação, colaboração e dar suporte a construir e compartilhar produtos digitais. Fornecer feedback durante o processo de construção das atividades educacionais, oportunizando aos alunos fazer perguntas e observações para acompanhamento. Possibilitar aos alunos discutir, concordar ou discordar dos comentários do professor durante o percurso (Ferguson et al., 2019);

- Integração de tecnologias digitais para o ensino universal: Desenvolver materiais adequados a necessidade dos estudantes, ensinar como estudar a distância, construir métodos de controle e avaliação. A comunicação entre professores, família, escolas e a comunidade é essencial para assegurar que as necessidades dos alunos serão entendidas e tratadas de forma adequada, através de pesquisa questionários e diagnóstico continuado (OECD, 2018a; Schleicher, 2016);

- Saber qual tecnologia utilizar de acordo com cada necessidade: Confrontar a utilidade de diversas tecnologias e os seus recursos utilizando-se de critérios, como adequação ao conteúdo e ambiente, confiabilidade, segurança, qualidade, design, performance, atratividade e interatividade (Schleicher, 2016);

- Trabalho e planejamento em equipe: Utilizar tecnologias digitais para trabalhar colaborativamente, desenvolvendo materiais didáticos através de uma rede online com outros professores. Trabalhar em parcerias estratégicas com outras instituições de ensino para aprender e refletir com outros professores, além de sistematizar e aprimorar suas práticas inovadoras (Schleicher, 2016; Paniagua e Istance, 2018);

- Uso de tecnologias digitais para ensino e aprendizado pessoal e coletivo: Apoiar o processo de transformação do pensamento do aluno em algo visível, oferecendo oportunidades de armazenar e processar informações, desenvolvendo espaços de comunicação e cooperação, dando oportunidades para feedback rápido (Schleicher, 2016; Ferguson et al., 2019);

- Utilizar abordagens pedagógicas inovadoras: Na matemática, a inovação necessita elevar o envolvimento dos estudantes e os resultados da aprendizagem, podendo usar pedagogias mais abertas, como a aprendizagem baseada em problemas. No ensino de idiomas, a pedagogia deve proporcionar a possibilidade de desenvolver resultados, podendo ser através de aprendizado baseado em tarefas e projetos, interligando a contextos de outras culturas (Paniagua e Istance, 2018);

- Utilizar métodos de avaliação inovadores: Os alunos devem poder expressar seu entendimento em distintos pontos e poder avaliar o trabalho um do outro. Utilizar avaliações abertas que possibilitem uma pluralidade nas respostas dos alunos, permitindo com que eles possam selecionar suas próprias ferramentas e formas de apresentar suas ideias. Fornecer feedback útil para o aluno utilizando-se de, por exemplo, avaliação por pares, autoavaliação e aprendizado baseado em projetos (Sharples et al., 2016; Ferguson et al., 2019). 


\subsection{Soft Skill Discente}

Os modelos de educação devem adequar-se para fornecer aos estudantes as habilidades necessárias para desenvolver um mundo mais justo e produtivo (WEF, 2020). As escolas devem promover o aprendizado de habilidades centradas no ser humano (soft skills) que possibilitem aos alunos moldar sociedades que sejam inclusivas e equitativas (OECD, 2019; WEF, 2020). A seguir são apresentadas as Soft Skills apontadas como necessárias aos estudantes na jornada rumo à Educação 4.0:

- Adaptabilidade: Capacidade de se adaptar a diversos tipos de funções, responsabilidades, agendas, modificar concepções, métodos, posições ou metas com base em novas informações (WEF, 2016a; OECD, 2018b; P21, 2019).

- Aprender a aprender: Capacidade do estudante de ter autonomia para distinguir e saber não somente o que, mas também como aprender ao longo da vida. Possibilita ao futuro trabalhador intercalar facilmente de uma ocupação para outra (OECD, 2018b; Paniagua e Istance, 2018).

- Comunicação: Encadear pensamentos e ideias de modo efetivo através de habilidades de comunicação oral, escrita e não verbal de diversas formas e contextos (WEF, 2016a; OECD, 2018b; P21, 2019).

- Consciência social e cultural: Capacidade de inter-relacionar-se com outras pessoas de forma social, cultural e ética (WEF, 2016a, 2020; OECD, 2018b).

- Criatividade: Capacidade de produzir ou descobrir algo novo, transformar cenários e inovar na forma de agir. (WEF, 2016b, 2020; OECD, 2018b; P21, 2019).

- Curiosidade: Desejo acentuado de ver, ouvir, relacionar-se, testar algo novo, inédito, inexplorado. Capacidade de imaginar e considerar formas novas e inovadoras de tratar os problemas (WEF, 2016a, 2020; OECD, 2018b).

- Empatia: Capacidade de enxergar o mundo com os olhos dos outros, de entender o mundo por intermédio das experiências dos outros e de perceber o mundo através de emoções (OECD, 2018b; WEF, 2020).

- Iniciativa: Capacidade de ir além do domínio comum de habilidades para descobrir e ampliar o próprio aprendizado e as possibilidades de adquirir experiência (WEF, 2016a; P21, 2019).

- Liderança: Capacidade de guiar e inspirar as pessoas para atingir um objetivo em comum (WEF, 2016a; P21, 2019).

- Pensamento crítico e analítico: Utilizar raciocínio (indutivo, dedutivo) de acordo com cada situação. Capacidade de utilizar o pensamento sistêmico. Analisar indícios, premissas e concepções. Interpretar informações e tirar conclusões baseadas na melhor análise (WEF, 2016b, 2020; OECD, 2018b; P21, 2019).

- Persistência: Capacidade de firmar desejo, esforço e de obstinar-se no desenvolvimento de uma tarefa ou objetivo (WEF, 2016a; OECD, 2018b).

- Resolução de Problemas: Resolver distintos problemas de forma inovadora. Identificar e fazer perguntas significativas que esclareçam diversas perspectivas levando a melhora das soluções (WEF, 2016a, 2020; OECD, 2018b; P21, 2019).

- Responsabilidade: Refletir e analisar seus atos conforme suas experiências e metas pessoais e sociais. Comportar-se de modo responsável, tendo em mente os desejos da sociedade em geral (OECD, 2018 b; Paniagua e Istance, 2018; P21, 2019).

- Trabalho em equipe: Respeitar as diferenças culturais e trabalhar com pessoas de origens sociais, culturais e valores diferentes. Adquirir responsabilidade compartilhada pelo trabalho e enaltecer as contribuições individuais realizadas por cada membro da equipe (WEF, 2016a, 2016b, 2020; P21, 2019). 


\subsection{Hard Skill Discente}

As Hard Skills discente envolvem os conhecimentos e habilidades técnicas que os estudantes precisam ser equipados para atuar em pedagogias e metodologias ativas como aprendizagem baseada em problemas ou projeto. A seguir são apresentadas as Hard Skills apontadas como necessárias aos estudantes na jornada rumo à Educação 4.0:

- Design de tecnologias digitais: Capacidade de escrever programas de computador para diversos fins, possibilitando a melhora do desempenho em áreas relacionadas a lógica matemática e raciocínio. Auxilia no desenvolvimento de relacionamentos favoráveis com a tecnologia, entendendo os princípios para gerenciar riscos e segurança digital (WEF, 2016b; OECD, 2017; Paniagua e Istance, 2018).

- Gestão de Pessoas: Motivar e guiar as pessoas durante o desenvolvendo alguma atividade, distinguindo e indicando quais as melhores pessoas para cada ação ou conjunto de ações (WEF, 2016b; OECD, 2017).

- Gestão de Qualidade: Realização de testes e revisões de produtos, serviços ou processos com o intuito de analisar a qualidade ou o desempenho de uma etapa ou do projeto por inteiro (WEF, 2016b; OECD, 2017).

- Gestão de Recursos Tecnológicos: Capacidade de selecionar e utilizar recursos tecnológicos conforme a necessidade. Utilizar apropriadamente as ferramentas necessárias para um trabalho ou aprendizado (WEF, 2016a, 2020; OECD, 2017).

- Gestão de Risco: Capacidade de analisar as incertezas e desenvolver ações de controle para mitigar os impactos de custo, prazo, qualidade e escopo dos projetos, ou constatar as oportunidades que alguns riscos podem causar (OECD, 2019).

- Gestão de Tempo: Capacidade de gerenciar o próprio tempoe da equipe de trabalho, através de uma melhor autonomia e flexibilidade de horários, transformando-se no principal gestor do seu aprendizado (OECD, 2017, 2018a).

- Gestão Financeira: Ter hábitos de mitigação de consumo excessivo, planejar melhor seu consumo e seu futuro de modo consciente, responsável e compreender o papel da economia na sociedade (WEF, 2016a, 2016b; OECD, 2017; P21, 2019).

- Pensamento Computacional: Capacidade de resolver problemas de maneira lógica, utilizando conceitos como abstração, modularização e recursividade (Paniagua; Istance, 2018; WEF, 2020).

- Solução de problemas de maneira criativa: Adotar diferentes perspectivas, examinar, desenvolver e avaliar alternativas diferentes na resolução de problemas. Utilizar pensamento divergente, convergente e inventividade ao formar as soluções (Sharples et al., 2016).

\subsection{Pedagogia}

Inovações na pedagogia, como qualquer outra inovação, utiliza as concepções, materiais ou práticas já existentes e as agrupa de novas formas a fim de solucionar problemas quando a prática utilizada atualmente não responde de forma adequada às necessidades (Peterson et al., 2018). Muitas das pedagogias conhecidas como inovadoras demandam um planejamento e uma programação em que os alunos exerçam um papel ativo no gerenciamento de seu aprendizado; espera-se que eles construam e fortaleçam hábitos referentes a metacognição sobre o entendimento do que já se sabe e o que ainda é necessário entender melhor. A seguir são apresentadas as pedagogias consideradas mais adequadas em possibilitar ao estudante um papel ativo na promoção e exercício das habilidades e atitudes necessárias para a jornada rumo à Educação 4.0:

- Abordagens de Inovação (Design Centrado no Humano, Design Thinking, etc.): Possibilita que os estudantes se habituem com o aprendizado autogerenciado. Partem 
de um desafio e projetado de modo que os estudantes vivenciem o processo de concepção e desenvolvimento da solução, ao invés de encontrar uma "resposta" pronta (Sharples et al., 2016; Peterson et al., 2018; WEF, 2020).

- Aprendizagem Baseada em Problemas: Possibilita aprimorar as habilidades de resolução de problemas cotidianos. Dá a oportunidade paras os alunos se sentirem responsáveis pelo seu aprendizado e promove o desenvolvimento de um senso mais forte de comunidade em sala de aula (OECD, 2018a; P21, 2019; WEF, 2020).

- Aprendizagem Baseada em Projetos: Oferta uma estrutura para atividades, através de dilemas reais ou simulados de determinadas organizações, permitindo que os estudantes se acostumem com práticas reais com a mentoria dos professores (WEF, 2017; OECD, 2018a; P21, 2019).

- Aprendizagem Lúdica (Gamificação, Serious Games): Aplica princípios de jogos em contex tos educacionais, adaptando elementos pedagó gicos para absorver parte do poder psicológico, emocional e social dos jogos. Proporciona engajamento, motivação e habilidades de criatividade, curiosidade e resolução de problemas. (OECD, 2018a; Peterson et al., 2018; WEF, 2020).

- Aprendizagem Orientada para a Ação: Possibilita que os alunos selecionem, planejem e implementem projetos sobre problemas sociais. Os alunos trabalham nos projetos de ação no ambiente escolar ou em atividades extracurriculares. Os alunos são instigados a refletir sobre o que atingiram, o aprendizado, e o que fariam de diferente em uma próxima oportunidade (Peterson et al., 2018).

- Blended Learning: Possibilita maximizar os benefícios da tecnologia e recursos digitais para melhorar o entendimento das instruções conforme cada necessidade individual, promovendo uma melhor interação entre estudantes e professores (Paniagua e Istance, 2018; WEF, 2020).

\section{Conclusões}

Para que se alcance a Educação 4.0, é essencial focar em como o conhecimento é repassado para os estudantes, e não em qual conteúdo é ensinado. Refere-se ao processo de ir além do conteúdo disciplinar e ensinar para os estudantes as habilidades apontadas como necessárias para o aprendizado e trabalho do século XXI. É essencial exercitar no estudante a capacidade de se adaptar, possibilitando que ele, conforme as necessidades, situações e circunstâncias, consiga superar os desafios impostos pela convivência em sociedade e pelo mercado de trabalho.

O espaço escolar terá que se adaptar para estar na vanguarda da educação com um fluxo contínuo e em constante expansão de informações, colaborações e inovações, transformando-se em ambientes de encontros presenciais e a distância, para disseminação de conhecimentos úteis e para promover habilidades aos estudantes através da prática. Nesse novo paradigma educacional, os professores terão que possuir competências para sustentar a busca pelo conhecimento ao longo da vida, para colaborar com os pares e desempenhar papeis de facilitadores e mentores do desenvolvimento dos estudantes.

Faz-se necessário estimular nos estudantes uma maior autonomia, autogestão e responsabilidade para que o aprendizado seja contínuo, dentro e fora do ambiente escolar, para que tenham a capacidade de aprender a aprender e se equiparem com as habilidades digitais exigidas pela $4^{a}$ Revolução Industrial. Para colocar isso prática, não basta somente disponibilizar infraestrutura e recursos tecnológicos, é necessária uma estratégia de transformação digital em direção à Educação 4.0, a qual enfatize a formação técnica de profissionais, mas também de cidadãos engajados para torn ar o mundo melhor.

Este trabalho apresenta como contribuição um panorama de habilitadores para nortear o desenho e a implementação de estratégias de tran sformação digital na educação. V. $18 \mathrm{~N}^{\circ} 1$, julho, 2020 RENOTE DOI: 
Os habilitadores foram levantados a partir da análise de relatórios e estudos publicados recentemente, basicamente pelas organizações internacionais UNESCO, OECD e WEF, que balizam o desenvolvimento de políticas educacionais em todo o mundo. Iniciativas de transformação digital na educação tendem a ser aceleradas após as consequências sociais e econômicas provocadas pela pandemia do COVID-19, a qual evidenciou que os sistemas educacionais não estão preparados para lidar como cenário de restrição de tempo e espaço físico. O problema se torna ainda mais preocupante em países como o Brasil, onde as desigualdades regionais e sociais amplificam as desigualdades educacionais .

A conclusão desse estudo é que a transformação digital em direção à Educação 4.0 deve tratar de maneira holística os seguintes fatores que serviram para categorizar os habilitadores: uso de tecnologias digitais, considerando as especificidades dos estudantes; adoção de processos e práticas organizacionais em alinhamento com as novas relações sociais e de trabalho; equipar professores com competências digitais (fazer e ser) para tratar com estudantes nativos digitais; equipar estudantes com as habilidades técnicas, cognitivas, sociais e emocionais dos estudantes necessárias para o aprendizado e trabalho do século XXI; e adoção de pedagogias inovadoras centradas na transferência e aquisição de conhecimento sob demanda para resolver um problema ou realizar uma tarefa.

\section{Referências Bibliográficas}

BAXENDALE, G. Digital Transformation isn't that Technical. ITNOW, v. 61, n. 2, p. 04-05, 2019.

FERGUSON, R.; COUGHLAN, T.; EGELANDSDAL， K.; GAVED, M.; HERODOTOU, C.; HILLAIRE, G.; JONES, D.; JOWERS, I.; KUKULSKA-HULME, A.; MCANDREW, P.; MISIEJUK, K.; NESS, I. J.; RIENTIES, B.; SCANLON, E.; SHARPLES, M.; WASSON, B.; WELLER, M.; WHITELOCK, D. Innovating Pedagogy 2019: Open University Innovation Report 7. Milton Keynes: Institute of Educational Technology, The Open University, 2019.

GIL, A. C. Como elaborar projetos de pesquisa. 4. ed. São Paulo: Atlas, 2007.

GLADWELL, M. The tipping point: How little things can make a big difference. Little, Brown, 2006.

HADDUD, A.; MCALLEN, D. Digital workplace management: exploring aspects related to culture, innovation, and leadership. In: 2018 Portland International Conference on Management of Engineering and Technology (PICMET). p. 1-6, IEEE, 2018.

KUKULSKA-HULME, A.;BEIRNE, E.; CONOLE, G.; COSTELLO, E.; COUGHLAN, T.; FERGUSON, R.; FITZGERALD, E.; GAVED, M.; HERODOTOU, C.; HOLMES, W.; MAC LOCHLAINN, C.; NIC GIOLLAMHICHIL, M.; RIENTIES, B.; SARGENT, J.; SCANLON, E.; SHARPLES, M.; WHITELOCK, D. Innovating Pedagogy 2020: Open University Innovation Report 8. Milton Keynes: Institute of Educational Technology, The Open University, 2020.

MINAYO, M. C. S.; DESLANDES, S. F.; CRUZ NETO, O.; GOMES, R. Pesquisa social: teoria, método e criatividade. In: Pesquisa social: teoria, método e criatividade. 2001.

OECD. Organisation for Economic Co-operation and Development. Coronavirus special edition: Back to school, Trends Shaping Education Spotlights. OECD Publishing, Paris. 2020.

OECD. Organisation for Economic Co-operation and Development. Getting Skills Right: Skills for Jobs Indicators, Getting Skills Right. OECD Publishing, Paris. 2017. 
OECD. Organisation for Economic Co-operation and Development. Going Digital: Shaping Policies, Improving Lives. OECD Publishing, Paris. 2019.

OECD. Organisation for Economic Co-operation and Development. Teaching for the future: Effective classroom practices to transform education. OECD, Publishing, Paris. 2018a.

OECD. Organisation for Economic Co-operation and Development. The future of education and skills: Education 2030. OECD Education 2030. OECD Publishing, Paris. $2018 b$.

P21. Partnership for 21 st Century Learning. Partnership for 21st Century Learning: Framework for 21st Century Learning Definitions. Battelle for Kids. 2019.

PANIAGUA, A.; ISTANCE, D. Teachers as Designers of Learning Environments: The Importance of Innovative Pedagogies. OECD Publishing, Paris. 2018.

PETERSON, A.; DUMONT, H.; LAFUENTE, M.; LAW, N. Understanding innovative pedagogies: Key themes to analyse new approaches to teaching and learning, OECD Education Working Papers, No. 172, OECD Publishing, Paris, 2018.

SCHLEICHER, A. Teaching Excellence through Professional Learning and Policy Reform: Lessons from Around the World, International Summit on the Teaching Profession. OECD Publishing, Paris. 2016.

SCHWAB, K. A Quarta Revolução Industrial (Edipro). São Paulo. 2016.

SHARPLES, M.; ROOCK, R.; FERGUSON, R.; GAVED, M.; HERODOTOU, C.; KOH, E.; KUKULSKA-HULME, A.; CHEE-KIT, L.; MCANDREW, P.; RIENTIES, B.; WELLER, M.; WONG, L. H. Innovating pedagogy 2016: Open University innovation report 5. Milton Keynes: Institute of Educational Technology, The Open University, 2016.

TAM, G.; EL-AZAR, D. 3 ways the coronavirus pandemic could reshape education. 2020. Disponível em https://www.weforum.org/agenda/2020/03/3-way s-coronavirus-isreshaping-education-and-what-changes-might-be-here-to-stay. Acesso em: 02 maio 2020.

UNESCO. United Nations Educational, Scientific and Cultural Organization. COVID19 Educational Disruption and Response. 2020. Disponível em https://pt.unesco.org/covid19/educationresponse. Acesso em: 02 maio de 2020.

UNESCO. United Nations Educational, Scientific and Cultural Organization. The futures of learning 2: What kind of learning for the 21st century. Education Research and Foresight Working Papers, v. 3, 2015.

WEF. World Economic Forum. New Vision for Education: Fostering Social and Emotional Learning through Technology. Switzerland. 2016a.

WEF. World Economic Forum. Schools of the Future. Defining New Models of Education for the Fourth Industrial Revolution. Switzerland. 2020.

WEF. World Economic Forum. Schools of the Future. Realizing Human Potential in the Fourth Industrial Revolution. An Agenda for Leaders to Shape the Future of Education, Gender and Work. Switzerland. 2017.

WEF. World Economic Forum. The future of jobs: Employment, skills and workforce strategy for the fourth industrial revolution. In: Global Challenge Insight Report, World Economic Forum, Geneva, 2016 b. 